\title{
Chemical Composition, Antioxidant and Antibacterial Activity of Different Extracts of Poplar Type Propolis
}

\author{
Tomislav Mašek, ${ }^{1}$ Nataša Perin, ${ }^{2}$ Livio Racané, ${ }^{3}$ Maja Cindrić, ${ }^{2}$ Hana Čipčić Paljetak, ${ }^{4}$ Mihaela Perić, ${ }^{4}$ Mario Matijašić, \\ Donatella Verbanac, ${ }^{4}$ Božo Radić, ${ }^{5}$ Jelena Šuran, ${ }^{6}$ Kristina Starčević $7, *$

\footnotetext{
1 Department of Animal Nutrition and Dietetics, Faculty of Veterinary Medicine, University of Zagreb, Heinzelova 55, HR-10000 Zagreb, Croatia

2 Department of Organic Chemistry, Faculty of Chemical Engineering and Technology, University of Zagreb, Marulićev trg 19, HR-10000 Zagreb, Croatia

${ }^{3}$ Department of Applied Chemistry, Faculty of Textile Technology, University of Zagreb, Prilaz baruna Filipovića 28a, HR-10000 Zagreb, Croatia

4 Division of Molecular Medicine, Laboratory for Epigenomics, School of Medicine, University of Zagreb, Šalata 2, HR-10000 Zagreb, Croatia

5 Hedera, d.o.o, 4. gardijske brigade 35, Kamen HR-21000 Split, Croatia

6 Department of Pharmacology, Faculty of Veterinary Medicine, University of Zagreb, Heinzelova 55, HR-10000 Zagreb, Croatia

7 Department of Animal Husbandry, Faculty of Veterinary Medicine, University of Zagreb, Heinzelova 55, HR-10000 Zagreb, Croatia

* Corresponding author's e-mail address: kristina.starcevic@vef.hr
}

RECEIVED: February 1, 2018 * REVISED: April 6, 2018 औ ACCEPTED: April 6, 2018

\begin{abstract}
Chemical composition, antioxidant activity and in vitro antibacterial activity of propolis from the central, continental part of Croatia were studied. Propolis hydro-ethanolic extracts (PHEE), prepared using three different methods and two solvent mixtures contained high amounts of flavonoids (20.95-28.11\% TIC), aromatic acids (8.17-15.91\% TIC) and their esters (9.27-11.91 \% TIC). The PHEE obtained in this study showed high antioxidant activity (DPPH IC 50 values from $9.96-19.95 \mu \mathrm{g} / \mathrm{ml}$ and FRAP $38.0-41.9 \mathrm{mM} \mathrm{Fe}{ }^{2+} / \mathrm{mg}$ PHEE). Despite differences in composition, the PHEE samples exhibited significant antibacterial activities, affecting tested strains of Staphylococcus aures, Enterococcus faecalis, Escherichia coli and Moraxella catarrhalis. The use of different solvent ratio and extraction procedures selectively increases or decreases the content of specific propolis components in the extract which can have a beneficial effect on the application of propolis extracts.
\end{abstract}

Keywords: Propolis, Antioxidant activity, Antibacterial agents.

\section{INTRODUCTION}

$\mathbf{P}$ ROPOLIS is one of the apicultural products that have been used from ancient times as natural remedy due to its beneficial influence on human health. ${ }^{[1-3]}$ During the past decades, propolis became widely accepted and commercially used in the form of home remedies, toothpastes, creams, ointments, drops, and dietary supplements.

Once collected, propolis is enriched with bee's salivary and enzymatic secretions. Propolis is used by bees as a natural sealer in hives as well as protective barrier against intruders. ${ }^{[4]}$ It is considered that propolis has important role in immunity of honey bees by reducing the spread of bacteria and parasites in hives and eliminating biological contamination in a colony. ${ }^{[5]}$ As a natural product, propolis has complex chemical composition and several types. The most investigated propolis type, poplar propolis, accounts for more of 300 identified compounds divided into: aromatic acid esters, terpenoids, aromatic acids, long-chain aliphatic fatty acids and their esters, aliphatic hydrocarbons and wax esters, amino acids, flavones and flavonols, aliphatic acids (short-chain), glycerol derivatives, flavanones, aldehydes, alcohols, aliphatic acid esters, chalcones, sugars and sugar alcohols, acetophenones and other ketones, dihydrochalcones, steroids and miscellaneous ingredients. ${ }^{[6,7]}$ The chemical composition of propolis is variable, depending on the 

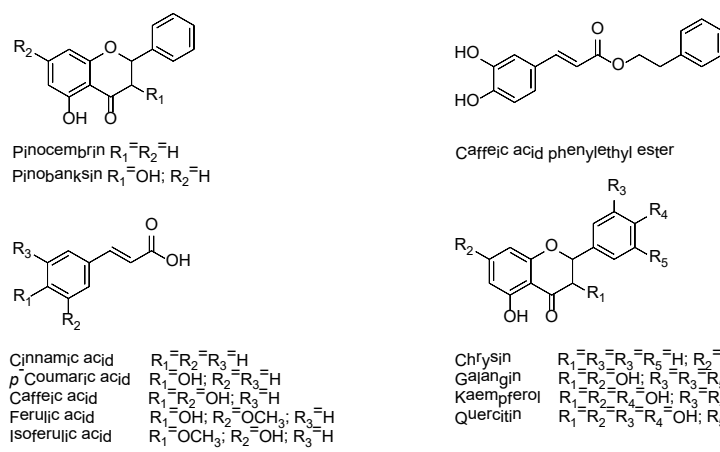

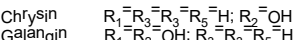
kaempferol $R_{1}=R_{2}=R_{2}=R_{3}: R_{3} R_{5}=$

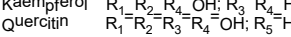

Figure 1. Some of representative phenolic compounds in propolis.

regional plant ecology. In regions with temperate climate, the propolis resin is collected mainly from the buds and cracks in the bark of Populus species. Resulting poplar type propolis is characterized with flavonoids without B-ring substituents, such as pinocembrin, pinobanksin, galangine and chrysin and phenylpropanoid acids and their esters, e.g. caffeic acid phenethyl ester (CAPE) (Figure 1). ${ }^{[8]}$

The bioactive substances in propolis are responsible for the broad spectrum of biological activities: antioxidant, ${ }^{[9]}$ antifungal, ${ }^{[10]}$ antibacterial, ${ }^{[11,12]}$ antitumor ${ }^{[13]}$ and anti-inflammatory. ${ }^{[14]}$ Some of the compounds of poplar type propolis gained specific interest in human and veterinary medicine in the last decades. Caffeic acid phenethyl ester (CAPE) has broad biological activities, including anti-inflammatory properties, inhibition of cell proliferation induction of cell cycle arrest and apoptosis. ${ }^{[14]}$ Flavonoids, particularly quercetin, kaempherol, apigenin and chrysin, ubiquitous in plants and present in the different human diets have antioxidative, hypolipidemic, antibacterial, antitumor, anti-inflammatory, antidiarrhoeal, antiulcer, antimutagenic, neuroprotective, cardioprotective, vasodilator, immunomodulator, antidiabetic and hepatoprotective activity. ${ }^{[15-20]}$ The complex biological activity of propolis is a reason why elucidation of the complex chemical composition remains in the focus of many research studies. Moreover, fast and accurate identification of biologically active natural compounds is becoming important feature in drug discovery as a source of potential active molecules. ${ }^{[21]}$

Raw propolis cannot be used without prior solvent extraction that removes waxes and other redundant ingredients and preserves bioactive compounds. Solvents usually used in the extraction procedure are methanol, ethanol, water or their mixtures. The extraction is performed using elevated temperature (reflux) or maceration for several hours at room temperature. Nowadays, use of microwave assisted extraction [22] is increasingly being used because it significantly reduces the consumption of solvents and the time of extraction. Since biological activity of natural compounds depends on their chemical properties, we investigated the influence of different solvent ratios and extraction methods for obtaining propolis extracts with desirable composition and advantageous antioxidative and antibacterial activity.

\section{EXPERIMENTAL PART Reagents and Chemicals}

Bis(trimethylsilyl)trifluoroacetamide (BSTFA), analytical grade ethanol, cinnamic acid, $p$-coumaric acid, kaempferol, chrysin, caffeic acid, ferulic acid, quercetin, D-fructose, Dglucose, sucrose, palmitic acid, stearic acid, apigenin, Folin-Ciocalteu reagent, Trolox (6-hydroxy-2,5,7,8-tetramethylchroman-2-carboxylic acid), 1,1-diphenyl-2-picrylhydrazyl radical (DPPH), 2,4,6-tris(2-pyridyl)-s-triazine (TPTZ) and 2,2'-azinobis(3-ethylbenzothiazoline-6-sulfonic acid) (ABTS) were obtained from Aldrich (Steinheim, Germany).

\section{Propolis Collection and Extracts Preparation}

Propolis samples were obtained from several locations throughout the central, continental Croatia during 2014. Crude propolis samples were frozen $\left(-20^{\circ} \mathrm{C}\right)$ until extract preparation.

Propolis was grounded in a chilled grinder and small amounts $(0.5 \mathrm{~g})$ of pulverised crude propolis were extracted with $10 \mathrm{ml}$ of EtOH- $\mathrm{H}_{2} \mathrm{O}$ in two ratios 70:30 and 80:20 $(\mathrm{v} / \mathrm{v})$. The obtained mixtures were stirred at room

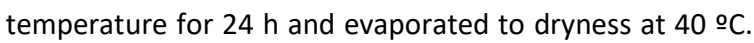
Upon filtration and evaporation concentrated products were frozen at $-20 \stackrel{\circ}{ } \mathrm{C}$ and freeze dried. The yields obtained for different ratios of EtOH- $\mathrm{H}_{2} \mathrm{O}:$ 70:30 and 80:20 $(\mathrm{v} / \mathrm{v})$, were 65.80 and $77.80 \%$, respectively.

The extraction was performed by refluxing a weighed amount of sample $(0.5 \mathrm{~g})$ with $10 \mathrm{ml}$ of solvent at two different $\mathrm{EtOH}-\mathrm{H}_{2} \mathrm{O}$ ratios, 70:30 and 80:20 ( $\left.v / v\right)$, for 24 $\mathrm{h}$ under stirring in water bath. Then, the obtained extracts were filtered, solvent evaporated to dryness, frozen at -20 o $\mathrm{C}$ and freeze dried. The yields obtained for $\mathrm{EtOH}-\mathrm{H}_{2} \mathrm{O}$ ratios 70:30 and 80:20 (v/v) were 66.60 and $65.40 \%$, respectively.

A weighed amount of grounded propolis $(0.5 \mathrm{~g})$ was extracted with $10 \mathrm{ml}$ of solvent at two different $\mathrm{EtOH}-\mathrm{H}_{2} \mathrm{O}$ ratios, 70:30 and 80:20 $(\mathrm{v} / \mathrm{v})$, in a $25 \mathrm{ml}$ glass vessel using microwave apparatus with closed vessel (Start Synth Milestone Inc. USA). The microwave experimental conditions were set as follows: extraction temperature 120 ${ }^{\circ} \mathrm{C}$ and extraction time $15 \mathrm{~min}$. The pressure was dynamically adjusted by temperature and power feedback control. During the extraction, magnetic stirring was 
applied to homogenize extraction solution. Afterword,

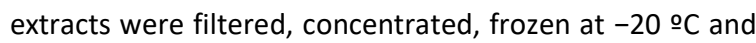
freeze dried. The yields obtained for $\mathrm{EtOH}: \mathrm{H}_{2} \mathrm{O}$ ratios $70: 30$ and $80: 20(v / v)$ were 49.80 and $56.20 \%$, respectively.

\section{Derivatisation and GC-MS Analysis of Propolis Extracts}

All samples of propolis extracts were derivatised prior to GC-MS analysis. For this purpose, propolis extracts were evaporated to dryness under nitrogen and $1 \mathrm{mg}$ of each dry extract was derivatised by the addition of $50 \mu$ l pyridine and $100 \mu \mathrm{l}$ bis-(trimethylsilyl)trifluoroacetamide (BSTFA) containing $1 \%$ trimethylchlorosilane (TMCS). Reaction proceeded in a sealed glass tube for $30 \mathrm{~min}$ at $70^{\circ} \mathrm{C}$.

All samples were analysed by Shimadzu GC-MS Ultra Gas Chromatograph Mass Spectrometer (Shimadzu, Kyoto, Japan), equipped with capillary column InertCap $1 \mathrm{MS}$ ( 0.25 $\mathrm{mm}$ internal diameter, $0.25 \mu \mathrm{m}$ film thickness, $30 \mathrm{~m}$ long, GL Sciences, Tokyo, Japan). The injector temperature was set to $250^{\circ} \mathrm{C}$, and $1 \mu \mathrm{l}$ of each sample was injected with a split ratio of 1:80. Helium was used as the carrier gas, and linear velocity was $35 \mathrm{~cm} / \mathrm{s}$. The oven program was set as follows: temperature at $100^{\circ} \mathrm{C}$ for $2 \mathrm{~min}$, then increased at the rate of $5{ }^{\circ} \mathrm{C} / \mathrm{min}$ up to $280{ }^{\circ} \mathrm{C}$ held $15 \mathrm{~min}$, then increased at $10^{\circ} \mathrm{C} / \mathrm{min}$ up to $320^{\circ} \mathrm{C}$ and held $20 \mathrm{~min}$. Mass selective (MS) detector operated under electron impact ionisation $(70 \mathrm{eV}$ ) and MS scan range was $35-1000 \mathrm{~m} / \mathrm{z}$. The interface and ion source temperatures were set at 280 and $250{ }^{\circ} \mathrm{C}$, respectively.

Peaks were identified by using mass spectra libraries (NIST 08 and Wiley 7). All the experimental measurements were repeated three times and the average values reported. Additionally, compounds were identified by comparing their mass spectra and characteristic ions of their TMS derivatives with the data available in literature: pinobanksin, and pinobanksin- $O$-acetate, ${ }^{[23]}$ anthraquinones, ${ }^{[24]}$ caffeic acid ester ${ }^{[25]}$ and chalcones. ${ }^{[26]}$ Cinnamic acid, $p$-coumaric acid, kaempferol, chrysin, caffeic acid, ferulic acid and quercetin were identified and quantified using standard solutions of the specific compound.

\section{Determination of Ferric Reducing/ Antioxidant Power (FRAP assay)}

The FRAP method was conducted according to previously reported procedure ${ }^{[27]}$ with minor assay modifications to a 96-well plate format. A solution of $10 \mathrm{mM}$ TPTZ and $20 \mathrm{mM}$ ferric chloride was diluted in $300 \mathrm{mM}$ sodium acetate buffer $(\mathrm{pH} 3.6)$ at a ratio of 1:1:10. The tested propolis extracts $(20 \mu \mathrm{l})$ were added to the 96-well microplate followed by working FRAP solution $(280 \mu \mathrm{l})$. The mixture was shaken and incubated $30 \mathrm{~min}$ at $37{ }^{\circ} \mathrm{C}$ in the dark. Final concentration of tested propolis extracts was $1 \mathrm{mg} / \mathrm{ml}$. The absorbance at $593 \mathrm{~nm}$ was recorded using microplate reader $\mu$ Quant (Biotec Inc.). For FRAP assay ferrous sulfate $\left(\mathrm{FeSO}_{4} \times 7 \mathrm{H}_{2} \mathrm{O}\right)$ was used to develop a 20-2000 $\mu \mathrm{mol} / \mathrm{L}$ standard curve. All results were then expressed as $\mathrm{Fe}^{2+}$ equivalents $\left(\mathrm{Fe}^{2+} \mathrm{mmol} / \mathrm{mg} \mathrm{PHEE}\right)$. All tests were done in triplicate and the results were averaged.

\section{DPPH Radical Scavenging Assay}

The determination of reducing activity was measured according to previously reported procedure with some modifications. ${ }^{[28]}$ Briefly, equal volume of tested propolis extracts at various concentrations was added to a solution of DPPH (final concentration $100 \mu \mathrm{M}$ in absolute ethanol). The assay was carried out in a 96 well microtiter plate. Ethanol was used as a negative control solution and solution of $10 \mathrm{mg} / \mathrm{ml}$ of $\alpha$-tocopherol was used as positive control. After $30 \mathrm{~min}$ in dark, the absorbance was recorded at $517 \mathrm{~nm}$ on $\mu$ Quant (Biotec Inc.) reader at room temperature. All measures were done in triplicate.

The percentage scavenging of test samples at each concentration were calculated using the following formula:

$$
\left[\left(\mathrm{Abs}_{\text {control }}-\mathrm{Abs}_{\text {compound }}\right) / \mathrm{Abs}_{\text {control }}\right] \times 100
$$

The $I_{50}$ values for each compound were calculated from dose-response curves using linear regression analysis.

\section{Determination of Total Phenolic Content}

The total phenolic content of propolis extracts was analyzed using gallic acid as a standard by the FolinCiocalteu method ${ }^{[29]}$ with some assay modifications to a $96-$ well plate format. Ethanol solutions of propolis extracts ( 2.5 $\mathrm{mg} / \mathrm{mL}$ ) were prepared and $20 \mu \mathrm{L}$ of these solutions or of gallic acid standard solutions or ethanol as blank were added to each well of the microplate. To each well, $20 \mu \mathrm{L}$ of Folin-Ciocalteu reagent (diluted in water 1:1) was added and incubated for 30 minutes at room temperature. Finally, $80 \mu \mathrm{L}$ of $7.5 \% \mathrm{Na}_{2} \mathrm{CO}_{3}$ solution was added, mixed by pipetting, plate was covered and incubated at room temperature for 2 hours. The absorbance was read at 725 $\mathrm{nm}$ using a microplate reader ( $\mu$ Quant, Biotec Inc.). The total phenolic content of the extracts was determined by comparison with a calibration curve of gallic acid standard and represented as $\mathrm{mg}$ gallic acid equivalents (GAE) in $\mathrm{g}$ of propolis extract. The analyses were done in triplicate.

\section{Antibacterial Activity}

Minimum inhibitory concentrations (MICs) were determined by the broth microdilution method according to guidelines of the Clinical Laboratory Standards Institute. ${ }^{[30]}$ Double dilutions of tested compounds and extracts in 96-well microtiter plates were prepared in $0.512-0.016 \mathrm{mg} / \mathrm{ml}$ concentration range and tested in duplicates. E.coli ECM1556 (efflux pump deficient strain, 
tolC:Tn10) and S. aureus (ATCC 29213) were grown on Mueller-Hinton agar plates (by Becton Dickinson, USA) and E. faecalis (ATCC29212) and M. catarrhalis (ATCC 23246) where grown on Columbia agar with $5 \%$ sheep blood. Bacterial inocula were prepared by direct colony suspension method and plates inoculated with $5 \times 10^{4}$ cfu/well. Results were determined by visual read-out after overnight incubation at $37{ }^{\circ} \mathrm{C}$ in ambient air. The ethanol:water solutions (70:30 and 80:20, v/v) were used as a negative control while azithromycin was used as a positive control antibiotic.

\section{Statistical Analysis}

Data were analyzed using the Statistica 12 program (STATISTICA 12 program, Tulsa, OK, USA). Normality of distribution was tested with Shapiro-Wilks test. All data are presented as means \pm standard deviation (SD). The data were tested by One-way ANOVA and post hoc Tukey test. The significance was set at $P<0.05$. Multi-variate principal component analysis (PCA) was used to relate GC-MS data and parameters of antioxidative status.

\section{RESULTS AND DISCUSSION}

\section{Chemical Composition of Propolis Hydro-Ethanolic Extracts (PHEE)}

The propolis was collected from the central, continental part of Croatia and analyzed by GC-MS (Figure 2). The results obtained by GC-MS analyses revealed that the hydroalcoholic extracts of Croatian propolis contained more than 50 compounds and 28 of them were identified. The chemical composition of PHEE as \% of TIC were presented and summarized in Table 1. Currently, there is limited literature data regarding the composition and biological properties of propolis from the central part of Croatia. ${ }^{[18,20,31]}$

The analytical results presented in Table 1 demonstrate that propolis from the central part of Croatia contains high concentration of flavonoids and phenolic acids such as ferrulic, $p$-coumaric and caffeic acid, benzyl caffeate, phenylethyl ester of caffeic acid (CAPE), and chrysin. Many studies confirmed that propolis from temperate climate zone, such as propolis from Croatia, originate mainly from exudates of Populus species and their hybrids, and are rich in flavonoids, phenolic acids and their esters.

In addition to the influence of geographic location, pronounced impact on chemical composition and biopolyphenol content is achieved by the solvent and the extraction method. This study examined the differences between conventional extraction methods such as maceration and heat reflux extraction, and microwave assisted extraction (MAE). ${ }^{[22]}$ The MAE represents reliable

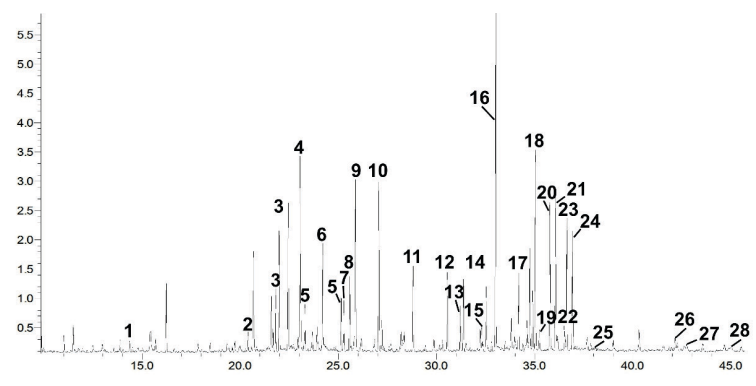

Figure 2. Representative chromatogram of propolis ethanolic extract. The numbers of compounds identified correspond to those in Table 1.

alternative to conventional techniques and has merits such as shorter time of extraction, less solvent consumption and higher yields of individual polyphenolic substances. ${ }^{[32] ~ I n ~}$ this study, two solvent ratios of ethanol and water, 80:20 and 70:30, respectively, were investigated. The analytical results clearly established that extraction methods and solvent ratios significantly influence quantity of individual compound as well as groups of active compounds with similar structure.

Overall, aromatic acids and their esters comprised 8.17-15.91 and 9.27-11.91\% of TIC, respectively. The most abundant aromatic acids were $p$-coumaric, ferrulic and caffeic acid. The highest content of aromatic acids was observed when maceration and 80:20 solvent ratio was applied (15.91\% TIC). Irrespective of the method used, 80:20 solvent ratio extracted more aromatic acids than 70:30 solvent ratio implying that when a high concentration of aromatic acids is needed in an extract, solvent ratio of $80: 20$ should be used. These aromatic acids possess significant antibacterial, antiinflammatory, hepatoprotective and antioxidant activity, ${ }^{[33]}$ therefore, their presence in the PHEE studied is beneficial for such applications. The esters of aromatic acids represent important group of polyphenolic compounds in propolis, with phenylethyl caffeate (CAPE) as the most investigated, due to its strong antitumor and antioxidant activity. ${ }^{[33]}$ Therefore, the high content of CAPE in studied extracts $(2.59-3.34 \% \mathrm{TIC})$ is considered biologically important. Based on the obtained results the MAE and solvent ratio $80: 20$ is a method of choice in order to extract higher content of CAPE.

All PHEE extracts contained flavonoids (flavanones, flavones and flavonols) at high level. The content of flavonoids was the highest in all PHEE (20.95-28.11\% TIC) irrespective of the extraction procedure or solvent ratio. The experimental data presented revealed that extract with the highest content of flavonoids could be obtained by MAE and solvent ratio 80:20 rather than with other methods. 
Table 1. Chemical composition of different propolis extracts assessed by GC-MS as trimethylsilyl ethers derivatives (\% of total ion current)

\begin{tabular}{|c|c|c|c|c|c|c|c|c|}
\hline \multirow{3}{*}{ No } & \multirow{3}{*}{ Compound } & \multirow{3}{*}{ Target ions } & \multicolumn{6}{|c|}{ Composition } \\
\hline & & & \multicolumn{2}{|c|}{ Maceration } & \multicolumn{2}{|l|}{ Reflux } & \multicolumn{2}{|c|}{ Microwave } \\
\hline & & & $70: 30^{\mathrm{a}}$ & $80: 20$ & $70: 30$ & $80: 20$ & $70: 30$ & $80: 20$ \\
\hline & Aromatic acids & & & & & & & \\
\hline $1^{\mathrm{b}}$ & trans-cinnamic & 131, 103, 205 & 0.19 & 0.22 & 0.12 & 0.14 & 0.15 & 0.16 \\
\hline $2^{c}$ & 4-methoxycinnamic acid & $161,191,235$ & 0.42 & 0.41 & 0.30 & 0.32 & 0.31 & 0.36 \\
\hline $4^{b}$ & p-coumaric acid & $73,219,293$ & 3.23 & 4.40 & 2.34 & 2.67 & 2.28 & 2.80 \\
\hline $6^{c}$ & 3,4-dymethoxycinnamic acid & $191,265,280$ & 2.11 & 2.26 & 1.28 & 1.45 & 1.45 & 1.83 \\
\hline $8^{c}$ & trans-isoferulic acid & $73,338,308$ & 1.32 & 1.62 & 0.80 & 0.84 & 0.82 & 1.06 \\
\hline $9^{b}$ & Ferulic acid & $73,338,249$ & 3.17 & 3.71 & 2.01 & 2.05 & 2.05 & 2.28 \\
\hline \multirow[t]{3}{*}{$10^{b}$} & Caffeic acid & 73, 219, 396 & 2.40 & 3.30 & 1.32 & 1.35 & 1.32 & 1.64 \\
\hline & $\Sigma$ aromatic acids & & 12.84 & 15.91 & 8.17 & 8.81 & 8.38 & 10.11 \\
\hline & Esters & & & & & & & \\
\hline $12^{d}$ & 3-methyl-3-buthenyl caffeate & $73,219,392,191$ & 1.71 & 1.58 & 1.36 & 1.22 & 1.37 & 1.68 \\
\hline $13^{d}$ & 2-methyl-2-buthenyl caffeate & $73,219,294$ & 0.94 & 0.85 & 0.83 & 0.72 & 0.77 & 1.01 \\
\hline $14^{d}$ & 3-methyl-2-buthenyl caffeate & $73,219,392,324$ & 1.78 & 1.55 & 1.90 & 1.68 & 1.88 & 2.23 \\
\hline $20^{d}$ & Benzyl caffeate & $73,91,414$ & 3.49 & 3.06 & 2.98 & 2.93 & 3.17 & 3.65 \\
\hline \multirow[t]{3}{*}{$24^{d}$} & Phenylethyl caffeate & $73,105,428$ & 3.05 & 2.59 & 2.75 & 2.72 & 2.87 & 3.34 \\
\hline & $\Sigma$ esters & & 10.97 & 9.62 & 9.83 & 9.27 & 10.06 & 11.91 \\
\hline & Sugars & & & & & & & \\
\hline $3^{b}$ & D-fructose & $73,204,147$ & 3.32 & 4.25 & 1.65 & 2.00 & 2.01 & 2.36 \\
\hline $5^{b}$ & D-glucose & 73, 204, 191 & 1.75 & 2.03 & 0.87 & 0.94 & 0.96 & 1.15 \\
\hline \multirow[t]{3}{*}{$22^{b}$} & Sucrose & $73,361,217$ & 0.20 & 0.47 & 0.40 & 0.41 & 0.44 & 0.53 \\
\hline & $\Sigma$ Sugars & & 5.27 & 6.75 & 2.92 & 3.35 & 3.40 & 4.04 \\
\hline & Fatty acids & & & & & & & \\
\hline $7^{b}$ & Palmitic acid & $73,117,75,313$ & 0.59 & 1.04 & 1.11 & 1.23 & 0.95 & 0.69 \\
\hline \multirow[t]{3}{*}{$11^{b}$} & Stearic acid & $73,117,75,341$ & 0.77 & 1.70 & 2.18 & 1.67 & 1.71 & 0.96 \\
\hline & $\Sigma$ fatty acids & & 1.37 & 2.74 & 3.28 & 2.90 & 2.66 & 1.65 \\
\hline & Flavanones & & & & & & & \\
\hline $15^{d}$ & Pinostrobin & $327,73,238$ & 0.90 & 0.51 & 0.67 & 0.88 & 0.83 & 1.17 \\
\hline $16^{d}$ & Pinocembrin & $73,385,296$ & 9.32 & 7.07 & 8.37 & 7.56 & 9.41 & 9.31 \\
\hline $17^{d}$ & Pinobanksin & 73, 296, 192 & 1.76 & 1.58 & 1.52 & 1.61 & 1.39 & 1.90 \\
\hline $18^{d}$ & Pinobanksin 3 acetate & $73,296,443$ & 5.68 & 4.20 & 5.08 & 4.69 & 5.04 & 5.94 \\
\hline $23^{c}$ & Galangin & $471,399,73$ & 3.51 & 2.66 & 3.14 & 2.87 & 3.54 & 3.64 \\
\hline \multirow[t]{3}{*}{$25^{d}$} & Naringenin & $73,473,296$ & 0.00 & 0.10 & 0.15 & 0.20 & 0.10 & 0.00 \\
\hline & $\Sigma$ flavanones & & 21.17 & 16.12 & 18.93 & 17.81 & 20.31 & 21.96 \\
\hline & Flavones and flavonols & & & & & & & \\
\hline $19^{d}$ & Tectochrysin & $325,155,282$ & 0.86 & 0.53 & 0.69 & 0.43 & 0.90 & 0.60 \\
\hline $21^{b}$ & Chrysin & $383,73,311$ & 5.42 & 3.45 & 4.15 & 3.25 & 5.38 & 4.64 \\
\hline $26^{b}$ & Kaempferol & $559,73,560$ & 0.35 & 0.43 & 0.39 & 0.33 & 0.36 & 0.44 \\
\hline $27^{b}$ & Apigenin & $471,73,472$ & 0.16 & 0.27 & 0.24 & 0.18 & 0.24 & 0.30 \\
\hline \multirow[t]{2}{*}{$28^{b}$} & Quercetin & $647,559,575$ & 0.00 & 0.15 & 0.15 & 0.11 & 0.15 & 0.17 \\
\hline & $\Sigma$ Flavones and flavonols & & 6.79 & 4.83 & 5.62 & 4.3 & 7.03 & 6.15 \\
\hline
\end{tabular}

aEthanol:water ratio

Methods of identification: bauthenitic standards, cmass spectral libraries (Wiley 7 and NIST 08), diterature

\section{PHEE Antioxidative Capacity and Total Phenolic Compounds}

The total polyphenol content and antioxidant activity of the PHEE extracts studied are presented in Figure 3 . In general, the increasing ratio of ethanol in the solvent mixture extracts higher quantities of phenolic compounds.

The total phenolic compounds (TPC) values ranged from 141.34 to $215.79 \mathrm{mg}$ GAE/g PHEE. These values were in range with previous reports for propolis from different parts of the world. ${ }^{[34]}$ The highest TPC value was observed for PHEE obtained by solvent reflux using solvent mixture $80: 20, v / v$ mixture. 

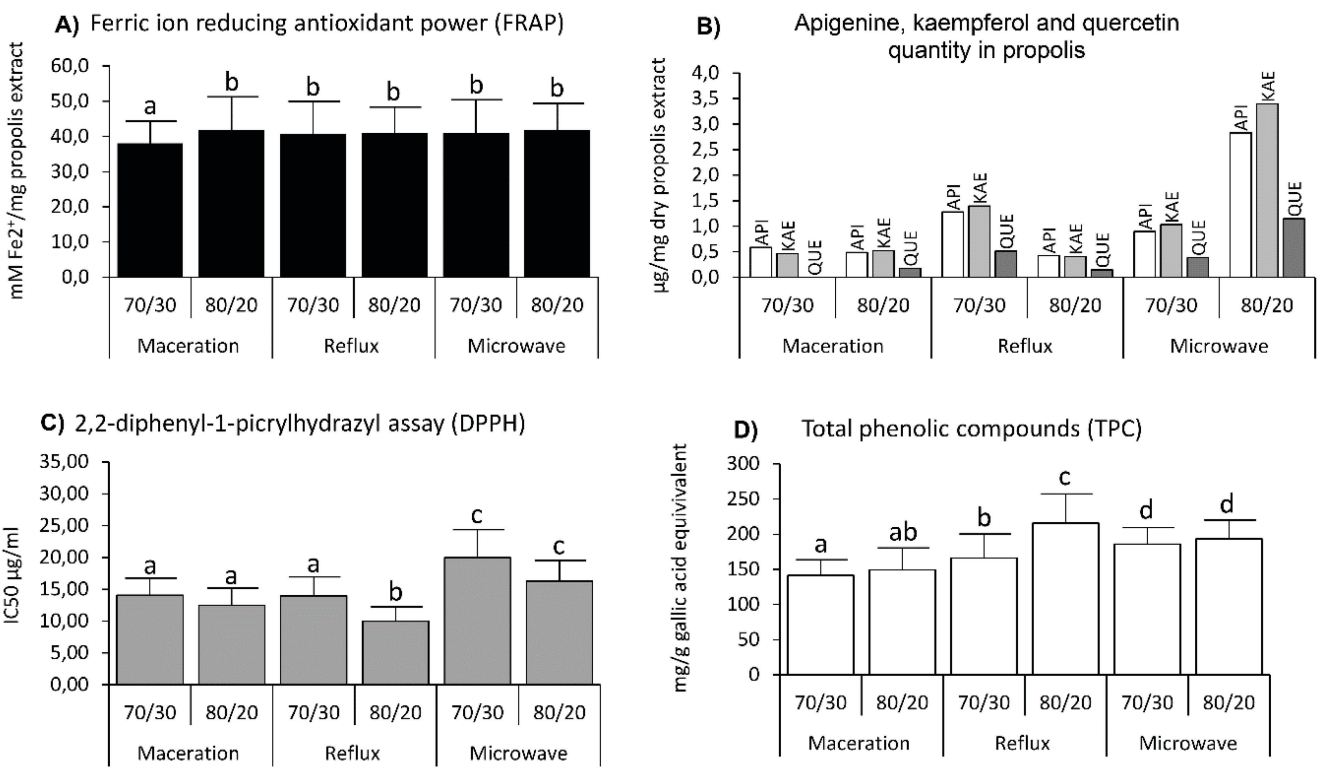

Figure 3. A) Antioxidant properties of different extracts measured as FRAP, B) Quantity of apigenine (API), kaempferol (KAE) and quercetin (QUE) in propolis extracts, C) Antioxidant properties of different extracts measured as DPPH and D) Total phenolic compounds measured as gallic acid equivivalent. Bars with different superscripts (a-d) differ significantly $(P<0.05)$.

As phenolic compounds in propolis play a protective role against oxidative damage caused by free radicals, we evaluated antioxidant properties of propolis extracts using two assays, DPPH and FRAP. The DPPH, a free radical that accepts an electron or hydrogen radical to become a stable diamagnetic molecule, is readily used to evaluate the antioxidant activity of propolis extracts. FRAP assay is based on reduction of TPTZ-Fe(III) complex to TPTZ-Fe(II) complex. Propolis ethanol extracts evaluated in this study showed high antioxidant activity (DPPH IC 50 values from 9.96 to $19.96 \mu \mathrm{g} / \mathrm{ml}$, FRAP $38.0-41.9 \mathrm{mM} \mathrm{Fe}{ }^{2+} / \mathrm{mg}$ PHEE). DPPH activity was mainly related to total phenolic compounds as shown in Figure $3 \mathrm{C}$ and D. Among identified PHEE components, quercetin, apigenin and kaempferol, showed correlation with FRAP assay (Figure 4). From the obtained results it is obvious that the increase of these three compounds, quercetin in particular, is responsible for antioxidant activity in FRAP assay (Figure 4). These compounds belong to flavonoids which antioxidant activity depends upon the arrangement of functional groups about the nuclear structure. The configuration, substitution, and total number of hydroxyl groups mediate their antioxidant effects by scavenging free radicals and/or by chelating metal ions. [35]

\section{Chemometric Approach - Principal Component Analysis}

In order to analyse the large amount of analytical data we applied chemometric approach: the Principal Component
Analysis (PCA). The central idea of the PCA is to reduce the dimensionality of a data set in which there is a large number of correlated variables, while retaining the total information as much as possible. Chemometric studies

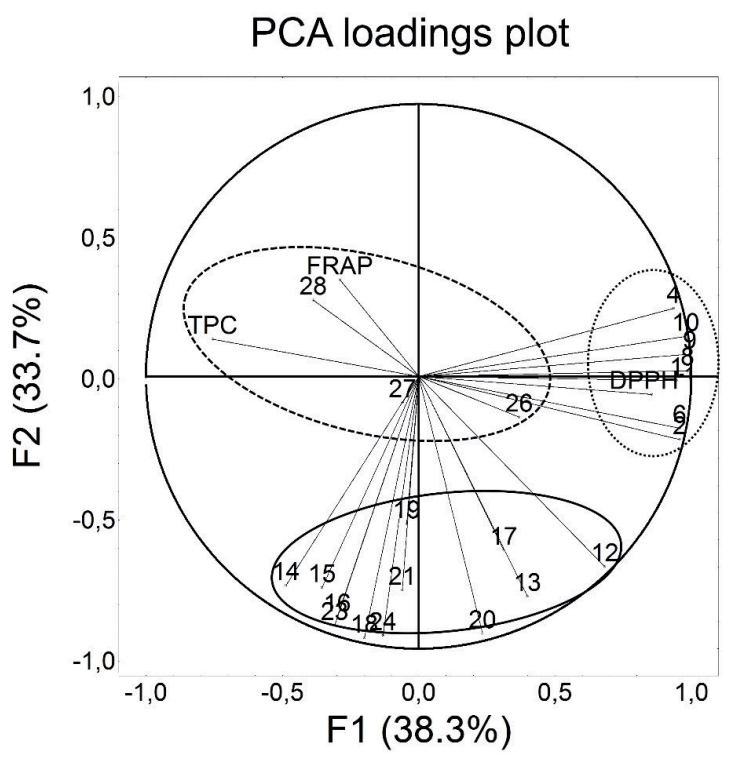

Figure 4. PCA loadings plot. The PCA loading plot was generated using parameters obtained by GC-MS and using the parameters of antioxidative properties. The numbers correspond to those in Table 1 
Table 2. Minimum inhibitory concentration (MIC, mg/ml) of PHEE towards selected strains

\begin{tabular}{|c|c|c|c|c|c|c|}
\hline & \multicolumn{2}{|c|}{ Maceration } & \multicolumn{2}{|l|}{ Reflux } & \multicolumn{2}{|c|}{ Microvawe } \\
\hline & $70: 30^{a}$ & $80: 20$ & $70: 30$ & $80: 20$ & $70: 30$ & $80: 20$ \\
\hline S. aureus ATCC 13709 & 0.128 & 0.128 & 0.128 & 0.128 & 0.128 & 0.128 \\
\hline M. catarrhalis ATCC 23246 & 0.016 & 0.016 & 0.016 & 0.016 & 0.032 & 0.016 \\
\hline E. faecalis ATCC29212 & 0.256 & 0.256 & 0.256 & 0.256 & 0.256 & 0.256 \\
\hline E. coli TolC- & 0.032 & 0.064 & 0.064 & 0.064 & 0.032 & 0.032 \\
\hline
\end{tabular}

aEthanol:water ratio.

were undertaken to explore possible variations among PHEE. Figure 4. represents a PCA loadings plot constructed using the relative amounts of individual constituents of propolis from the GC-MS analysis (Table 1.) and values obtained for TPC, FRAP and DPPH.

PCA loadings plot showed high relation between the results from DPPH assay and the amount of aromatic acids while FRAP values were mostly related to the measured quantities of quercetin, apigenin and kaempferol. Other propolis components' amounts were not related to parameters of antioxidative properties.

\section{Antibacterial Activity}

The antibacterial activity of propolis is a result of different propolis constituents as well as their synergistic effects. ${ }^{[36]}$ All PHEE exhibited some antibacterial effects towards tested bacterial strains and presented in Table 2. There was no difference among PHEE extracts in their antibacterial activity against $S$. aureus and E. feacalis and MICs were $0.128 \mathrm{mg} / \mathrm{ml}$ and $0.256 \mathrm{mg} / \mathrm{ml}$, respectively. These values are comparable to those obtained for poplar type propolis. ${ }^{[37]}$

In contrast to previous results which did not find antibacterial activity of poplar type propolis against tested gram negative bacteria ${ }^{[38]}$ our results showed notable activity of Croatian propolis against tested strains of $M$. catarrhalis and E. coli with MICs values $0.016-0.032$ $\mathrm{mg} / \mathrm{ml}$ and $0.032-0.064 \mathrm{mg} / \mathrm{ml}$, respectively. The antibacterial activity of propolis is of great importance for the bee community and propolis always possesses some degree of antibacterial activity. ${ }^{[39]}$ Nevertheless no single propolis component has been shown to possess antibacterial activity higher than the complex extract. ${ }^{[40,41]}$ Therefore the quantification of the active compounds into groups having the same or close chemical structure correlates better with the biological activity. ${ }^{\left[{ }^{[8]}\right.}$ The biological activity of propolis component is mainly attributed to a few classes such as flavonoids, phenolic acids and their esters and terpenes. Determined chemical composition (Table 1) indicates that considerable amount of flavonoids and esters of phenolic acids is present in propolis which is generally regarded to be responsible for the antibacterial activity of propolis.

\section{CONCLUSION}

In conclusion, Croatian propolis extracts are typical poplar type propolis rich in aromatic acids and flavonoids with very low content of terpenes. Even minor changes in solvent ratio, as well as extraction procedures significantly affect abundance of specific propolis components, and therefore, these changes could be used to selectively increase or decrease the content of specific components of interest and to obtain more standardized propolis extracts for use in human and veterinary medicine. The three extraction methods differed mainly in the total percentage of aromatic acids and flavonoids extracted, with maceration giving the highest quantity of aromatic acids, while microwave assisted extraction led to the extraction of the highest amount of flavonoids. Despite differences in chemical composition of different extracts, the PHEE studied showed no clear connection between chemical composition and biological activity. The obtained results indicate that the FRAP value is mostly related to the quercetin concentration, nevertheless, overall antioxidant capacity of propolis is the result of synergistic activity of individual phenolic acids and flavonoids.

Acknowledgment. This study was supported by the European Regional Development Fund and EU Structural Funds in the project: "Intramammary propolis formulation for prevention and treatment of mastitis in dairy ruminants".

\section{REFERENCES}

[1] A. Salatino, C. C. Fernandes-Silva, A. A. Righi, M. L. F.Salatino, Nat. Prod. Rep. 2011, 28, 925.

[2] A. K. Kuropatnicki, E. Szliszka, M. Klosek, W. Krol, Evid. Based Complement. Alternat. Med. 2013, Article ID 983974

[3] A. K. Kuropatnicki, E. Szliszka, W. Krol, Evid. Based Complement. Alternat. Med. 2013, Article ID 964149

[4] G. A. Burdock, Food Chem. Toxicol. 1998, 36, 347

[5] M. Simone-Finstrom, M. Spivak, Apidologie 2010, 41, 295. 
[6] A. C. De Groot, M. P. Popova, V. S. Bankova, An Update on the Constituents of Poplar-Type Propolis, AC Degroot Publishing, Wapserveen, Netherlands, 2014.

[7] C. Sun, Z. Wu, Z. Wang, H. Zhang, Evid. Based Complement. Alternat. Med. 2015, Article ID 595393

[8] M. Popova, V. Bankova, D. Butovska, V. Petkov, B. Nikolova-Damyanova, A. G. Sabatini, G. L. Marcazzan, S. Bogdanov, Phytochem. Anal. 2004, 15, 235.

[9] N. Kalogeropoulos, S. J. Konteles, E. Troullidou, I. Mourtzinos, V. T. Karathanos, Food Chem. 2009, 116, 452.

[10] A. B. S. Siqueira, B. S. Gomes, I. Cambuim, R. Maia, S. Abreu, C. M. Souza-Motta, L. A. de Queiroz, A. L. F. Porto, Lett. Appl. Microbiol. 2009, 48, 90.

[11] M. L. Castro, J. A. Cury, P. L. Rosalen, S. M. Alencar, M. Ikegaki, S. Duarte, H. Koo, Quim. Nova, 2007, 30, 1512.

[12] S. M. Alencar, T. L. C. Oldoni, M. L. Castro, I. S. R. Cabral, C. M. Costa-Neto, J. A. Cury, P. L. Rosalen, M. Ikegaki, J. Ethnopharmacol. 2007, 113, 278.

[13] S. Khacha-Ananda, K. Tragoolpua, P. Chantawannakul, Y. Tragoolpua, Asian Pac. J. Cancer Prev. 2013, 14, 6991.

[14] N. Marquez, R. Sancho, A. Macho, M. A. Calzado, B. L. Fiebich, E. Munoz, J. Pharmacol. Exp. Ther. 2004, 308, 993.

[15] M. A. Lea, C. Ibeh, J. K. Deutsch, I. Hamid, C. Desbordes, Anticancer Res. 2010, 30, 3629.

[16] H. Sies, T. Schewe, Y. Steffen, C. Heis, M. Kelm, Free Radical Res. 2005, 39, S22-S22.

[17] H. Oksuz, N. Duran, C. Tamer, M. Cetin, S. Silici, Ophthalmic Res., 2005, 37, 328.

[18] N. Oršolić, V. Benković, A. Horvat-Knezević, N. Kopjar, I. Kosalec, M. Bakmaz, Z. Mihaljević, K. Bendelja, I. Bašić, Biol. Pharm. Bull. 2007, 30, 946.

[19] L. V. Peng-Cheng, K. R. Wang, Q. S. Li, J. Chen, J. Sun, H. L. Zhu, Bioorg. Med. Chem. 2010, 18, 1117.

[20] N. Oršolić, G. Gajski, V. Garaj-Vrhovac, D. Dikić, Z. S. Prskalo, D. Sirovina, Eur. J. Pharmacol. 2011, 656, 110.

[21] F. Li, S. Awale, Y. Tezuka, S. Kadota, Bioorg. Med. Chem. 2008, 16, 5434.

[22] J. M. Thevelein, R. Gelade, I. Holsbeeks, O. Lagatie, Y. Popova, F. Rolland, F. Stolz, S. Van de Velde, P. Van Dijck, P. Vandormael, A. Van Nuland, K. Van Roey, G. Van Zeebroeck, B. Yan, Biochem. Soc. Trans. 2005, 33, 253.
[23] M. Neacsu, P. C. Eklund, R. E. Sjoholm, S. P. Pietarinen, M. O. Ahotupa, B. R. Holmbom, S. M. Willfor, Holz Roh-Werkst. 2007, 65, 1.

[24] Y. Zuo, C. Wang, Y. Lin, J. Guo, Y. Deng, J. Chromatogr. A 2008, 1200, 43.

[25] W. Greenaway, E. Wollenweber, T. Scaysbrook, F. R. Whatley, Z. Naturforsch., C: J. Biosci. 1988, 43, 795.

[26] W. Greenaway, F.R. Whatley, J. Chromatogr. A 1990, 519, 145.

[27] I. F. F. Benzie, J. J. Strain, Anal. Biochem. 1996, 239, 70.

[28] D. Valencia, E. Alday, R. Robles-Zepeda, A. GaribayEscobar, J. C. Galvez-Ruiz, M. Salas-Reyes, M. Jimenez-Estrada, E. Velazquez-Contreras, J. Hernandez, C. Velazquez, Food Chem. 2012, 131, 645-651.

[29] V. L. Singleton, R. Orthofer, R. M. Lamuela-Raventos, Methods Enzymol. 1999, 299, 152.

[30] CLSI document M07-A8, Methods for Dilution Antimicrobial Susceptibility Tests for Bacteria That Grow Aerobically; Approved Standard-Eighth Edition, Clinical and Laboratory Standards Institute, Wayne, PA, USA, 2009.

[31] I. Jerković, Z. Marijanović, P. M. Kus, C. I. G. Tuberoso, Chem. Biodiversity 2016, 13, 210.

[32] W. Routray, V. Orsat, Food Bioprocess Technol. 2012, 5, 409.

[33] V. Bankova, J. Ethnopharmacol. 2005, 100, 114.

[34] S. Kumazawa, H. Goto, T. Hamasaka, S. Fukumoto, T. Fujimoto, T. Nakayama, Biosci. Biotechnol. Biochem. 2004, 68, 260.

[35] K. E. Heim, A. R. Tagliaferro, D. J. Bobilya, J. Nutr. Biochem. 2002, 13, 572.

[36] E. L. Ghisalberti, Bee World 1979, 60, 59.

[37] G. Vardar-Unlu, S. Silici, M. Unlu, World J. Microbiol. Biotechnol. 2008, 24, 1011.

[38] S. Silici, S. Kutluca, J. Ethnopharmacol. 2005, 99, 69.

[39] V. Bankova, R. Christov, A. Kujumgiev, M. C. Marcucci, S. Popova, Z. Naturforsch., C: J. Biosci. 1995, 50, 167.

[40] J. S. Bonvehi, F. V. Coll, R. E. Jorda, J. Am. Oil Chem. Soc. 1994, 71, 529.

[41] A. Kujumgiev, I. Tsvetkova, Y. Serkedjieva, V. Bankova, R. Christov, S. Popova, J. Ethnopharmacol. 1999, 64, 235. 